\title{
Restaurações cerâmicas multicamadas e monolíticas: uma revisão de literatura
}

\section{Multilayered and monolithic ceramic restorations: a literature review}

\author{
Wilson da Silva Junior" \\ Priscilla do Monte Ribeiro Busato* \\ Marcio José Mendonça ${ }^{* * *}$ \\ Veridiana Camilotti**** \\ Juliana Aparecida Delben ${ }^{* * * *}$
}

\section{Resumo}

Objetivo: realizar uma revisão de literatura sobre materiais, técnicas de confecção, vantagens, desvantagens e limitações das restaurações cerâmicas multicamadas e monolíticas. Revisão de literatura: embora as cerâmicas odontológicas sejam utilizadas para confecção de restaurações dentárias indiretas, porque têm excelentes propriedades mecânicas e estéticas, são materiais susceptíveis à fratura sob forças oclusais. Para compensar essa fragilidade, restaurações multicamadas são confeccionadas associando um material mais resistente para a infraestrutura e a posterior aplicação de cerâmica para recobrimento estético. Porém, as restaurações multicamadas apresentam como desvantagem o possível lascamento da cerâmica de recobrimento. Essas falhas comprometem a restauração funcional e a estética, exigindo a substituição da peça protética em muitos casos. Sendo assim, devido à evolução de sistemas cerâmicos e técnicas de processamento, surgiram as restaurações cerâmicas monolíticas fabricadas com um único material, a fim de superar tal limitação. Nesse sentido, diferentes materiais e técnicas são apresentados na literatura para a confecção de restaurações cerâmicas monolíticas. Considerações finais: as restaurações multicamadas têm bom resultado estético, porém podem apresentar lascamento da cerâmica de recobrimento. Essa limitação é superada pelo uso de um único material para as restaurações monolíticas, mas o resultado estético pode ser limitado. De um modo geral, a literatura demonstra um bom comportamento clínico para as restaurações cerâmicas multicamadas e monolíticas, quando a técnica de confecção e o sistema cerâmico são bem indicados.

Palavras-chave: Cerâmica. Prótese dentária. Prótese parcial fixa.

\section{Introdução}

As cerâmicas odontológicas são materiais utilizados para restaurações dentárias indiretas, como coroas, inlays, onlays e facetas. ${ }^{1}$ Esse material possui excelentes propriedades, tais como biocompatibilidade, estabilidade química, baixa condutividade térmica, alta resistência à compressão, difusividade térmica, translucidez, fluorescência e coeficiente de expansão térmica semelhante ao da estrutura dentária. No entanto, a cerâmica é um material frágil, devido à presença de microfissuras em sua superfície, tornando-a suscetível à fratura durante a cimentação e sob forças oclusais. ${ }^{2}$

Para compensar essa fragilidade, restaurações multicamadas associando a cerâmica a uma infraestrutura metálica vêm sendo utilizadas na Odontologia por aproximadamente 50 anos. ${ }^{2}$ Contudo, o aumento da demanda por restaurações dentárias estéticas culminou com o desenvolvimento de restaurações totalmente cerâmicas por meio da substituição da infraestrutura metálica. ${ }^{3}$

As restaurações multicamadas passaram, então, a ser confeccionadas com sistemas metal-free, utilizando diferentes materiais cerâmicos para infraestrutura e recobrimento estético. ${ }^{4}$ Porém, as restaurações multicamadas apresentam como desvantagens o risco de lascamento da cerâmica de cobertura devido a alguns fatores, como menor resistência à fratura da cerâmica de cobertura comparada com o material de infraestrutura, ${ }^{5}$ menor

Mestrando em Odontologia, Universidade Estadual do Oeste do Paraná, Departamento de Odontologia, Cascavel, PR, Brasil.

Pós-doutoramento em Odontologia, Universidade Estadual do Oeste do Paraná, Departamento de Odontologia, Cascavel, PR, Brasil.

Doutor em Materiais Dentários, Universidade Estadual do Oeste do Paraná, Departamento de Odontologia, Cascavel, PR, Brasil.

Doutora em Materiais Dentários, Universidade Estadual do Oeste do Paraná, Departamento de Odontologia, Cascavel, PR, Brasil.

Doutora em Prótese Dentária, Universidade Estadual do Oeste do Paraná, Departamento de Odontologia, Cascavel, PR, Brasil. 
espessura da camada de infraestrutura ${ }^{6}$ presença de defeitos na cerâmica de cobertura ou na interface infraestrutura/cobertura ${ }^{7}$ estresse residual, que surge da incompatibilidade do coeficiente de expansão térmica linear e da presença de tensão devido ao processo de resfriamento, ${ }^{8,9}$ entre a cerâmica de infraestrutura e a cobertura. Essas falhas comprometem a restauração funcional e a estética, exigindo a substituição da restauração, quando a área fraturada é muito extensa. ${ }^{10}$ Nesse sentido, diante da evolução dos sistemas cerâmicos e do avanço das técnicas de confecção, surgiram as restaurações cerâmicas monolíticas, com a finalidade de superar esse problema. Esse novo sistema tem excelentes propriedades mecânicas, além de propriedades estéticas satisfatórias. ${ }^{7}$

Entre as cerâmicas odontológicas, a cerâmica feldspática é muito utilizada devido à sua alta translucidez, que proporciona uma fiel mimetização do esmalte natural. No entanto, devido a sua baixa resistência mecânica, é indicada como recobrimento em restaurações multicamadas e restaurações cerâmicas parciais. ${ }^{11}$ Tentando contornar esse problema, surgiram as cerâmicas reforçadas por meio da adição de leucita, dissilicato de lítio, silicato de lítio reforçado com zircônia, alumina e zircônia. No entanto, resistência mecânica e propriedades estéticas são indiretamente proporcionais, e, por isso, as indicações desses materiais acabam sendo limitadas. $^{7}$

Diante desses fatores, é necessário que o profissional conheça as indicações e as características das cerâmicas odontológicas, a fim de determinar a classe apropriada de cerâmica para o uso em caso específico. Ao entender as classificações e a composição dos sistemas cerâmicos mais recentes, os profissionais podem determinar o material e a técnica de confecção ideal para cada tratamento. ${ }^{12}$

Portanto, os objetivos desta revisão de literatura são apresentar e discutir materiais, técnicas de confecção, vantagens, desvantagens e limitações das restaurações cerâmicas multicamadas e monolíticas.

\section{Materiais e método}

A pesquisa foi realizada nas bases de dados SciELO e PubMed, utilizando as seguintes palavras-chave: cerâmica, prótese dentária, prótese parcial fixa, monolithic restorations, multilayered dental prostheses e processing technologies. Foram selecionados ao todo 61 artigos, sendo 57 em língua inglesa e 4 em língua portuguesa, de acordo com a relevância para o tema. O período de pesquisa englobou publicações de 2000 a 2018 .

\section{Revisão da literatura}

\section{Restaurações multicamadas}

As restaurações multicamadas consistem em restaurações fabricadas com dois tipos de materiais, sendo um para a infraestrutura e outro para o revestimento. ${ }^{13}$ Tradicionalmente, essas restaurações eram confeccionadas com uma infraestrutura metálica e uma camada de cerâmica feldspática, sendo consideradas o padrão-ouro na Odontologia, devido ao seu excelente desempenho clínico. ${ }^{11}$ Estudos mostram taxa de falha anual do sistema metalocerâmico em torno de $1 \%$ e taxa de sobrevivência de $94 \%$ após 5 anos. ${ }^{14}$ A principal desvantagem do sistema metalocerâmico é a limitação estética, pois o cooping metálico é opaco e reflete a luz, produzindo uma aparência artificial da restauração. ${ }^{15}$ Devido à presença de duas camadas (metal e cerâmica), o desgaste do remanescente dental também acaba sendo maior durante o preparo protético. Além disso, a falta de adesão do metal ao agente cimentante e ao substrato dental exige que o preparo protético seja levemente retentivo, para auxiliar o embricamento mecânico do cimento e evitar o descolamento da peça. ${ }^{14}$

A crescente demanda por procedimentos estéticos culminou com a evolução e a criação de novos sistemas cerâmicos..$^{16}$ A cerâmica feldspática foi a precursora dos diversos tipos de cerâmicas encontradas atualmente. Como principais vantagens, apresenta alta translucidez e boa aparência estética, criando a ilusão de dentes naturais. No entanto, esse material tem baixa resistência mecânica, com resistência à flexão de 46,4 a 66,7 MPa. Além disso, tem alto coeficiente de contração, gera maior propagação de trincas, possui menor resistência à compressão e causa maior desgaste do antagonista. ${ }^{12}$

A busca por restaurações cerâmicas estéticas, substituindo o uso do metal e ampliando a indicação para dentes posteriores, exigiu a melhoria das suas propriedades mecânicas. Isso aconteceu com o aumento da fase cristalina por meio da adição de diversos componentes à cerâmica feldspática tradicional, criando, assim, as cerâmicas reforçadas. Os cristais atuam como bloqueadores da propagação de fendas, quando a cerâmica é submetida a tensões de tração, aumentando a resistência do material. ${ }^{17-19}$

Um material cerâmico amplamente utilizado para a confecção de infraestrutura é a zircônia tetragonal estabilizada com ítria (Y-TZP), pois apresenta propriedades mecânicas superiores às outras cerâmicas disponíveis (resistência à flexão $=800$ $1000 \mathrm{MPa}$; tenacidade à fratura $=6-8 \mathrm{MPa} \cdot \mathrm{m}^{1 / 2}$; dureza Vickers $=98,1 \mathrm{~N}$ ). No entanto, como a zircônia é um material extremamente opaco, o resultado estético é obtido por meio da aplicação da cerâmica de cobertura.$^{20}$ Porém, falhas podem ocorrer durante a vida útil dessas restaurações, como, por exemplo, o 
lascamento ou a delaminação da cerâmica de cobertura e/ou a fratura da infraestrutura..$^{13}$

O recobrimento da infraestrutura de zircônia com uma cerâmica de cobertura torna a restauração multicamada menos resistente e com maior taxa de insucesso, em comparação com as restaurações monolíticas. Isso provavelmente acontece devido a alguns fatores. ${ }^{7} \mathrm{O}$ primeiro fator é que a cerâmica de cobertura está suscetível à falha, quando submetida a menor carga, por apresentar menor resistência à fratura do que o material de infraestrutura. Embora sejam resistentes sob força de compressão, os materiais cerâmicos são frágeis e incapazes de resistir às tensões de tração complexas que ocorrem durante o carregamento funcional. ${ }^{5} \mathrm{O}$ segundo fator é que o uso da cerâmica de cobertura para melhorar a estética frequentemente requer uma redução da espessura da infraestrutura, podendo limitar as propriedades mecânicas da restauração. ${ }^{6} \mathrm{O}$ terceiro fator é que a técnica convencional de aplicação da cerâmica de cobertura pode gerar defeitos dentro da cerâmica de cobertura ou na interface de infraestrutura/cobertura, devido à incorporação de bolhas, vazios e microfalhas no interior na restauração. ${ }^{7} \mathrm{O}$ quarto e último fator é que o estresse residual que surge da incompatibilidade do coeficiente de expansão térmica linear entre a cerâmica de infraestrutura e a cobertura e a presença de tensão devido ao processo de resfriamento aumentam significativamente a incidência de fraturas da cerâmica de cobertura. Isso ocorre devido às diferenças de propriedades térmicas que afetam a termodinâmica da cerâmica vítrea durante o resfriamento. ${ }^{8,9}$

Apesar de a cerâmica de cobertura ser utilizada primeiramente por razões estéticas, ela tem um papel importante no comportamento mecânico da restauração. A resistência flexural e a tenacidade à fratura das restaurações multicamadas dependem da camada de revestimento, pois a trinca começa nas superfícies dessa camada. Embora as tensões compressivas residuais na camada de revestimento aumentem a tensão flexural das restaurações bilaminadas, a tensão de tração é a principal causa de lascamento. ${ }^{9-21}$

Comparando os diferentes materiais para confecção de restaurações multicamadas, um estudo clínico randomizado e controlado não observou diferenças significantes na condição clínica de restaurações multicamadas de zircônia e ouro-cerâmica em dentes posteriores, por um período de 5 anos. ${ }^{22}$ Do mesmo modo, restaurações multicamadas de zircônia e metalocerâmica foram acompanhadas por um período de 3 anos, e os autores não observaram diferenças significantes nos desempenhos clínico e estético entre os dois sistemas. ${ }^{23}$

Já um estudo clínico de 6 anos sobre restaurações multicamadas cerâmicas e metalocerâmicas identificou taxa de sobrevivência significativamente menor para as restaurações metal-free. ${ }^{24}$ En- quanto as falhas mais comumente relacionadas com as restaurações metalocerâmicas estão associadas com fraturas ou cáries nos dentes pilares, ${ }^{25,26} \mathrm{a}$ complicação clínica mais comumente relatada na falha de restaurações totalmente cerâmicas é a fratura da cerâmica de cobertura e/ou da infraestrutura. ${ }^{4,10,27-32}$

De maneira geral, as taxas de sobrevivência global das restaurações cerâmicas multicamadas variam entre 88 e $100 \%$ após 2 anos em serviço $^{10,16,27,28,33-35}$ e entre 84 e $97 \%$ após 5 a 14 anos em função. ${ }^{31,35,36}$ Porém, existem diferenças nas taxas de sobrevivência observadas para essas restaurações, quando se avalia cada sistema cerâmico isoladamente. Um estudo clínico randomizado acompanhou restaurações multicamadas de zircônia confeccionadas com diferentes técnicas de cobertura (tradicional e injeção) durante 3 anos e obteve taxa de sobrevivência de $100 \%$, além de não apresentar diferença significativa no lascamento da camada de cobertura nas duas técnicas de recobrimento. ${ }^{37}$

Existem diversas técnicas para aplicação da cerâmica de cobertura sobre a infraestrutura, entre elas, as técnicas de estratificação, injeção ou computer aided design/computer aided machining (CAD-CAM). Todas essas técnicas visam a otimizar a resistência dessa camada e, em alguns casos, reduzir o estresse na geração de calor residual. ${ }^{7}$

$\mathrm{Na}$ técnica de estratificação, a cerâmica de cobertura é confeccionada pela aplicação de várias camadas de uma mistura contendo o pó de cerâmica e o líquido de modelagem (água destilada misturada com modificadores reológicos) com o uso de um pincel. Porém, essa técnica gera camadas de estratificação suscetíveis a porosidades e uma série de defeitos intrínsecos que podem atuar como áreas de concentração de estresse, favorecendo a fratura da restauração durante a mastigação. ${ }^{7,13,38,39}$

$\mathrm{Na}$ técnica de injeção, a cerâmica de revestimento é fornecida sob a forma de pastilha e injetada em um molde refratário contendo a infraestrutura previamente sinterizada. Essa técnica resulta em uma camada de revestimento com menor porosidade e melhor resistência mecânica em comparação a uma camada de estratificação aplicada pela técnica tradicional. ${ }^{7}$

Atualmente, a evolução da tecnologia CAD-CAM possibilitou a facilidade de confecção da infraestrutura cerâmica ou camada de cobertura pela técnica de fresagem. ${ }^{38,39}$ Uma grande vantagem desse sistema é que os blocos usados para produção da camada de revestimento são originados a partir de procedimentos otimizados de sinterização em que o fabricante utiliza condições industriais ideais, resultando em blocos mecanicamente mais fortes e com menos defeitos em comparação com as camadas de recobrimento obtidas pelas técnicas descritas anteriormente. ${ }^{7,38,39}$ 


\section{Restaurações monolíticas}

O avanço das técnicas de confecção das restaurações totalmente cerâmicas culminou com a criação das restaurações monolíticas, que são peças confeccionadas inteiramente com um único tipo de cerâmica. ${ }^{7}$ Nessa técnica, a camada de revestimento ou cobertura é eliminada, o que reduz o tempo de fabricação e aumenta consideravelmente a resistência ao lascamento e à fratura. Assim, não há mais problemas relacionados com a união entre as camadas, e a espessura do material que confere resistência fica maior, proporcionando desgastes dentários com finalidade protética mais conservadores. ${ }^{40}$

As restaurações cerâmicas monolíticas têm apresentado melhor desempenho em relação às restaurações cerâmicas multicamadas, suportando cargas oclusais mais altas. As coroas cerâmicas monolíticas têm menor incidência de fratura pelo fato de que, na sua composição, só está envolvido um material. Além disso, as coroas monolíticas oferecem tempo reduzido de confecção e melhor custo-benefício. ${ }^{41}$

A estética e a menor diversidade de cores para esse tipo de restauração estão entre as principais limitações. Inicialmente, as restaurações monolíticas são peças monocromáticas que necessitam de técnicas de coloração nas camadas superficiais, para conferir maior naturalidade. ${ }^{42}$ Entre essas técnicas, estão maquiagem, adição de pigmentos à composição da cerâmica, imersão do material sinterizado em uma solução contendo diversos tipos de corantes ou produção de blocos pré-sinterizados de cerâmica pré-colorida com uma tonalidade mais homogênea. Uma vez dominadas tais técnicas, é possível a obtenção de resultados surpreendentes, principalmente para a região anterior. ${ }^{7}$

As restaurações monolíticas podem ser confeccionadas utilizando cerâmicas vítreas (reforçadas com leucita e dissilicato de lítio), híbridas (silicato de lítio reforçado com zircônia) ou cristalinas (alumina ou zircônia). ${ }^{7}$

A cerâmica reforçada com leucita (IPS Empress $1{ }^{\circledR}$ CAD, Authentic $\AA$ e VITABLOCS $\AA$ Mark II) tem boa resistência mecânica, com resistência mecânica entre 97 e $180 \mathrm{MPa}$. Além disso, apresenta excelentes propriedades estéticas e, consequentemente, tem demonstrado sucesso clínico a longo prazo, em situações de estresse mais elevado ou quando há maior exposição de dentina. Esse material pode ser altamente translúcido, mas tradicionalmente exige dimensões ligeiramente mais espessas para a trabalhabilidade e a compatibilidade estética. ${ }^{11}$

Já a cerâmica reforçada com dissilicato de lítio (IPS Empress $2 \circledR$ - Ivoclar Vivadent) apresenta propriedades mecânicas superiores, com resistência que varia de 300 a $400 \mathrm{MPa}$. Esse material sofreu algumas modificações e deu origem ao sistema IPS e.max (Ivoclar Vivadent), que possui melhores propriedades mecânicas, principalmente devido à di- minuição do tamanho dos cristais conformados e ao aumento do intertravamento entre os cristais, inibindo assim a propagação de trincas. Esse material também possui excelente estética, devido à eficiente mimetização do esmalte natural. ${ }^{43,44}$

A evolução dos sistemas cerâmicos tem como finalidade suprimir as suas desvantagens remanescentes. Para isso, foi desenvolvida a vitrocerâmica reforçada com silicato de lítio e zircônia (Suprinity ${ }^{\circledR}$ - Vita Zahnfabrik e CELTRA Duo® - Dentisply/Sirona). ${ }^{7}$ Esse material possui silicato de lítio (principal fase cristalina) em uma matriz vítrea reforçada com cristais de dióxido de zircônio $(\sim 10 \%) .{ }^{43}$ Quando esse material passa pelo processo de cristalização, os cristais de silicato de lítio atingem um tamanho 6 vezes menor do que o observado para os cristais de dissilicato de lítio presentes nas cerâmicas reforçadas com dissilicato de lítio. ${ }^{44} \mathrm{~A}$ formação de uma fase cristalina menor e mais fina ocorre devido à presença de partículas de zircônia, atuando como um aditivo que influencia a cristalização e impede o crescimento de cristais. Uma microestrutura contendo cristais menores garante ao material boas propriedades mecânicas, semelhantes às observadas para cerâmicas de dissilicato de lítio. Devido à sua excelente qualidade estética, é uma alternativa válida para reabilitações protéticas com alta demanda estética. Além disso, os blocos dessa vitrocerâmica são fresados mais rápidos em máquinas CAD-CAM e apresentam polimento superior em função do tamanho menor de cristais. ${ }^{7}$

Já as cerâmicas cristalinas (alumina e zircônia) possuem alta resistência mecânica devido a sua mínima ou nenhuma fase vítrea, porém diferem das vitrocerâmicas pela maneira com que a matriz cristalina se une às partículas na fase cristalina. ${ }^{11}$ Para superar os problemas relacionados ao lascamento, foi desenvolvida a zircônia tetragonal estabilizada por ítria (Y-TZP) para restaurações monolíticas. O aumento da tenacidade à fratura da Y-TZP para restaurações monolíticas é consequência de um mecanismo de tenacificação relacionado à transformação dos cristais tetragonais na fase monoclínica, que gera tensões de compressão em torno de defeitos, dificultando a propagação catastrófica. Além disso, a melhor translucidez ocorre por causa da diminuição do teor de alumina, do aumento da densidade, da diminuição do tamanho dos cristais, da adição de zircônia cúbica e da diminuição da quantidade de impurezas e defeitos estruturais. ${ }^{7}$

Um estudo retrospectivo avaliou a falha em restaurações de dissilicato de lítio monolíticas e multicamadas. Foram avaliadas 5.802 restaurações monolíticas e 5.538 restaurações multicamadas, fabricadas por dois laboratórios comerciais em um período de 45 meses. As restaurações de dissilicato de lítio tiveram baixa taxa de fratura, sendo que as multicamadas apresentaram uma taxa de fratura duas vezes maior do que as monolíticas. ${ }^{42}$ 
Guess et al. ${ }^{45}$ (2010) avaliaram o comportamento à fadiga e a confiabilidade das restaurações monolíticas de dissilicato de lítio (IPS e.max) e zircônia estratificada manualmente (Zir-CAD/Ceram) por meio de testes de fadiga de contato deslizante por carga cíclica. Os testes de fadiga mostraram que a aplicação de dissilicato de lítio pelo CAD/CAM em configuração monolítica resultou em restaurações resistentes à fadiga, ao passo que a aplicação manual das camadas estratificadas nas coroas de zircônia revelou elevada suscetibilidade à carga cíclica, ocasionando falha prematura nas coroas estratificadas.

Do mesmo modo, diversos estudos ${ }^{16,42,45-47}$ denotam resultados favoráveis ao uso de restaurações cerâmicas monolíticas para modalidades de tratamento envolvendo coroas totais, facetas, próteses parciais fixas e abutments. $\mathrm{O}$ índice de delaminação é menor do que o das restaurações multicamadas. Apesar de as restaurações monolíticas não apresentarem excelência em detalhes óticos, como fluorescência, translucidez e personalizações, obtidas por meio da técnica de estratificação, as técnicas de maquiagem têm evoluído juntamente com as propriedades óticas dos blocos fresados, tornando os resultados bastante aceitáveis.

$\mathrm{Na}$ comparação da resistência à fratura das coroas monolíticas de dissilicato de lítio (IPS e.max) e zircônia com diferentes espessuras, os resultados mostraram que as coroas monolíticas de zircônia com 0,6 mm de espessura podem fornecer resistência à fratura semelhante à de uma coroa monolítica de dissilicato de lítio de $1,5 \mathrm{~mm} .{ }^{48}$ Outro estudo avaliou a resistência à fratura de coroas monolíticas com diferentes espessuras $(0,3 \mathrm{~mm}, 0,5 \mathrm{~mm}$, $0,7 \mathrm{~mm}, 1 \mathrm{~mm}$ e $1,5 \mathrm{~mm}$ ) e diferentes materiais (zircônia altamente translúcida, zircônia de baixa translucidez e dissilicato de lítio IPS e.max CAD) e concluiu que as coroas de zircônia apresentaram resistência à fratura semelhante entre si, independentemente das espessuras, e superior ao dissilicato de lítio.49

Estudos clínicos têm mostrado adequado comportamento clínico de restaurações monolíticas de zircônia em diferentes situações. Um exemplo é o estudo clínico de Moscovitch ${ }^{50}$ (2015), que acompanhou, por 68 meses, restaurações monolíticas de zircônia em dentes e sobre implantes. Para isso, foram avaliadas 1.022 restaurações nos seguintes parâmetros: fratura, rachaduras ou lascamentos da cerâmica. Observou-se que não houve complicações nas restaurações em relação aos itens avaliados, e a zircônia mostrou-se um material confiável para todas as aplicações clínicas avaliadas no estudo.

Uma questão adicional a ser considerada é a influência da dureza superficial das restaurações monolíticas sobre o desgaste da superfície dental e a dificuldade de ajuste oclusal. Amer et al. ${ }^{51}$ (2015) compararam a rugosidade da superfície oclusal ao esmalte dental, após ciclos mastigatórios repetitivos com três cerâmicas (Y-TZP, dissilicato de lítio e porcelana feldspática convencional de baixa fusão). Os autores verificaram que as superfícies da cerâmica monolítica de zircônia e de dissilicato de lítio não se tornaram tão rugosas quanto as da cerâmica feldspática convencional. Em concordância com esse achado, diversos estudos demonstraram que coroas monolíticas de dissilicato de lítio e zircônia desgastam menos o esmalte do dente antagonista do que cerâmicas feldspáticas ou o próprio esmalte. ${ }^{52-55}$

As restaurações monolíticas também podem ser confeccionadas por meio das técnicas de prensagem ou injeção de pastilhas e fresagem em CAD-CAM. ${ }^{7}$ A cerâmica mais utilizada para confecção das restaurações monolíticas pela técnica de injeção é a cerâmica de dissilicato de lítio que apresenta pastilhas com diferentes cores e translucidez. A principal vantagem dessa técnica é o menor custo laboratorial em comparação ao sistema CAD-CAM. ${ }^{56}$

Já o sistema CAD-CAM produz restaurações com melhor adaptação e menor tempo, devido ao fato de os blocos serem mecanicamente mais fortes e com menos defeitos estruturais. As cerâmicas utilizadas para confecção de restaurações monolíticas por meio do sistema CAD-CAM são as cerâmicas reforçadas com leucita, dissilicato de lítio, silicato de lítio reforçado com zircônia e Y-TZP. ${ }^{57,58}$

\section{Discussão}

As restaurações totalmente cerâmicas são a preferência de muitos profissionais e pacientes, em razão de sua alta capacidade de mimetizar os tecidos dentais. No entanto, as cerâmicas são frágeis e apresentam baixa resistência mecânica, quando submetidas às tensões de tração, o que compromete o desempenho clínico em alguns aspectos. ${ }^{2}$

Para contornar esse problema, desenvolveram-se materiais cerâmicos reforçados que têm maior resistência mecânica, devido ao aumento da fase cristalina. Contudo, resistência e translucidez são grandezas inversamente proporcionais. Nesse sentido, o aumento do conteúdo cristalino na composição das cerâmicas aumentou os valores de resistência à fratura, porém tornou os sistemas mais opacos ou menos translúcidos. Além disso, melhorias nas propriedades mecânicas também foram alcançadas pelo aprimoramento dos métodos de processamento. ${ }^{59}$

As restaurações cerâmicas multicamadas podem ser confeccionadas com uma infraestrutura de zircônia revestida com cerâmica de recobrimento estético. ${ }^{4} \mathrm{~A}$ infraestrutura apresenta estabilidade dimensional, resistência mecânica e módulo de elasticidade semelhante às ligas metálicas. ${ }^{7}$ Já o revestimento é confeccionado com cerâmica de alta translucidez e boa aparência estética, criando a ilusão de dentes naturais..$^{12}$ Porém, falhas podem ocorrer durante a vida útil dessas restaurações, como, por exemplo, o lascamento ou a delaminação da ce- 
râmica de cobertura e/ou a fratura da infraestrutura. $^{13}$

Um estudo in vitro de Quinn et al. ${ }^{60}$ (2010) observou resistência ao lascamento similar entre restaurações multicamadas com infraestrutura de zircônia e metálicas, apesar da grande diferença na dureza do substrato. Zhang et al. ${ }^{61}$ (2012) verificaram que as restaurações multicamadas com cobertura de zircônia infiltrada com vidro são mais resistentes às lascas do que as com cobertura de cerâmica feldspática e, pelo menos, tão resistentes quanto a zircônia não infiltrada.

$\mathrm{Na}$ tentativa de superar os problemas relacionados ao lascamento da camada de cobertura e devido ao avanço das técnicas de processamento, surgiram as restaurações monolíticas. ${ }^{7}$ Nesse sistema, a camada de cobertura é eliminada, o que reduz o tempo de fabricação e aumenta consideravelmente a resistência ao lascamento e à fratura. Assim, não há mais problemas relacionados com a união entre as camadas, e a espessura do material que confere resistência fica maior, proporcionando desgastes dentários com finalidade protética mais conservadora. ${ }^{40}$

As restaurações cerâmicas monolíticas têm apresentado melhor desempenho em relação às restaurações cerâmicas multicamadas. As coroas cerâmicas monolíticas têm menor incidência de fratura pelo fato de que, na sua composição, só está envolvido um material. Além disso, esse sistema oferece tempo reduzido de confecção e melhor custo-benefício. ${ }^{41}$ No entanto, a ausência da camada de cobertura com cerâmica feldspática e a homogeneidade da coloração da peça se caracterizam como uma limitação estética. Para contornar esse problema, é necessário a realização de técnicas de coloração/maquiagem da restauração após a confecção. ${ }^{42}$

Os materiais mais utilizados para confecção das restaurações monolíticas são dissilicato de lítio, silicato de lítio reforçado com zircônia e Y-TZP, que possuem propriedades mecânicas e estéticas satisfatórias. ${ }^{7} \mathrm{O}$ dissilicato de lítio (IPS e.max - Ivoclar Vivadent) tem excelentes propriedades mecânicas e excelente estética em função da sua mimetização eficiente do esmalte natural. ${ }^{43,44} \mathrm{Na}$ tentativa de suprimir as desvantagens remanescentes desse sistema cerâmico, o silicato de lítio reforçado com zircônia (Suprinity ${ }^{\circledR}$ - Vita Zahnfabrik e CELTRA Duo® - Dentisply/Sirona) apresenta cristais menores, para garantir excelentes propriedades mecânicas e estéticas, além de polimento superior. Já a Y-TZP para restaurações monolíticas apresenta desempenho superior entre as cerâmicas odontológicas, devido a alto nível de resistência - mais de $1000 \mathrm{MPa}$ $\square$ e tenacidade à fratura superior - de 4 a $5 \mathrm{MPa}$. $\mathrm{m}^{1 / 2}$. Além disso, melhor translucidez ocorre em razão da diminuição do teor de alumina, do aumento da densidade, da diminuição do tamanho dos cristais, da adição de zircônia cúbica e da diminuição da quantidade de impurezas e defeitos estruturais. As restaurações monolíticas podem ser confeccionadas pela técnica de prensagem ou injeção de pastilhas e de fresagem utilizando a tecnologia CAD-CAM. ${ }^{7}$

Diante disso, as restaurações monolíticas de cerâmica estão sendo cada vez mais utilizadas na rotina clínica odontológica. A evolução dos materiais, as técnicas de confecção e as inúmeras vantagens desse sistema refletem na substituição gradual das restaurações multicamadas. No entanto, é importante que o profissional avalie individualmente cada caso, para realizar a escolha do sistema cerâmico mais adequado disponível no mercado.

\section{Considerações finais}

As restaurações cerâmicas monolíticas têm mostrado melhor desempenho em relação às restaurações cerâmicas multicamadas, ao apresentarem menor incidência de fratura, por utilizarem apenas um material. Além disso, as coroas monolíticas oferecem tempo reduzido de confecção e melhor custo-benefício para o paciente. Portanto, é necessário que o profissional tenha conhecimento de materiais e sistemas cerâmicos atuais visando a indicar a opção protética mais adequada para cada caso.

\section{Abstract}

Objective: the aim of this study was to conduct a literature review about the materials, techniques, advantages, disadvantages and limitations of multilayer and monolitic ceramic restorations. Literature review: although dental ceramics have been used for fabrication of dental restorations due to its good esthetic and mechanical properties, it is a material prone to fracture under oclusal loading. To surpass such fragility, multilayer restorations are fabricated using a resistant framework material associated to esthetic veneering ceramic. However, multilayer restorations present risk to chipping of veneering ceramic. Those failures represent a functional and esthetic problem, leading to restoration replacement. So, the evolution of ceramic systems and techniques generated monolitic restorations using a single material. Several materials and techniques are presented in the literature for fabrication of monolitic ceramic restorations. Final considerations: multilayer restorations present good esthetic result but delamination of veneering ceramic can occur. Such limitation is solved using a single material in monolitic restorations, but esthetic result is limited. In general, literature shows appropriate clinical performance for both multilayer and monolitic ceramic restorations when technique and materials are well indicated.

Keywords: Ceramics. Dental prosthesis. Fixed partial denture. 


\section{Referências}

1. Duzyol M, Sagsoz O, Polat Sagsoz N, Akgul N, Yildiz M. The Effect of Surface Treatments on the Bond Strength Between CAD/CAM Blocks and Composite Resin. J. Prosthodont $2016 ; 25: 466-71$

2. Guarda GB, Correr AB, Gonçalves LS, Costa AR, Borges GA, Sinhoreti MA, et al. Effects of Surface Treatments, Thermocycling, and Cyclic Loading on the Bond Strength of a Resin Cement Bonded to a Lithium Disilicate Glass Ceramic. Oper Dent 2013; 38:208-17.

3. Hooshmand T, Rostami G, Behroozibakhsh M, Fatemi M, Keshvad A, van Noort R. Interfacial fracture toughness of different resin cements bonded to a lithium disilicate glass ceramic. J Dent 2012; 40:139-45.

4. Bindl A, Richter B, Mormann WH. Survival of ceramic computer-aided design/manufacturing crowns bonded to preparations with reduced macroretention geometry. Int $\mathrm{J}$ Prosthodont 2005; 18(3):219-24.

5. Zhao K, Pan Y, Guess PC, Zhang XP, Swain MV. Influence of veneer application on fracture behavior of lithium-disilicatebased ceramic crowns. Dent Mater 2012; 28(6):653-60.

6. Silva NR, Bonfante EA, Martins LM, Valverde GB, Thompson VP, Ferencz JL, et al. Reliability of reduced-thickness and thinly veneered lithium disilicate crowns. J Dent Res 2012; 91:305-10.

7. Silva LHD, Lima E, Miranda RBP, Favero SS, Lohbauer U, Cesar PF. Dental ceramics: a review of new materials and processing methods. Dent Mater 2017; 31:133-46.

8. Zahran M, El-Mowafy O, Tam L, Watson PA, Finer Y. Fracture strength and fatigue resistance of all-ceramic molar crowns manufactured with CAD/CAM technology. J Prosthodont 2008; 17:370-7.

9. Belli R, Petschelt A, Lohbauer U. Thermal-induced residual stresses affect the fractographic patterns of zirconia-veneer dental prostheses. J Mech Behav Biomed Mater 2013; 21:167-77.

10. Wolfart S, Bohlsen F, Wegner SM, Kern M. A preliminary prospective evaluation of all-ceramic crown-retained and inlay-retained fixed partial dentures. Int J Prosthodont 2005; 18(6):497-505

11. Gomes EA, Assunção WG, Rocha EP, Santos PH. Cerâmicas odontológicas: o estado atual. Cerâmica 2008; 54(331):31925.

12. McLaren EA, Figueira J. Updating Classifications of Ceramic Dental Materials: A guide to material selection. Ins Dent 2015 ; 30:48-53.

13. Sailer I, Pjetursson BE, Zwahlen M, Hammerle CH. A systematic review of the survival and complication rates of allceramic and metal-ceramic reconstructions after an observation period of at least 3 years. Part II: Fixed dental prostheses. Clin Oral Implants Res 2007; 18(3):86-96.

14. Pjetursson BE, Sailer I, Makarov NA, Zwahlen M, Thoma DS. All-ceramic or metal-ceramic tooth-supported fixed dental prostheses (FDPs)? A systematic review of the survival and complication rates. Partm: multiple-unit FDPs. Dent Mater 2015; 31(6):624-39.

15. Smielak B, Klimek L. Effect of hydrofluoric acid concentration and etching duration on select surface roughness parameters for zirconia. J Prosthet Dent 2015; 13:596-602.

16. Raptis NV, Michalakis KX, Hirayama H. Optical behavior of current ceramic systems. Int J Periodontics Restorative Dent 2006; 26(1):31-41.
17. Anusavice KJ, Kakar K, Ferree N. Which mechanical and physical testing methods are relevant for predicting the clinical performance of ceramic-based dental prostheses? Clin Oral Implants Res 2007; 18:218-31.

18. Heintze SD, Cavalleri A, Forjanic M, Zellweger G, Rousson V. Wear of ceramic and antagonist - a systematic evaluation of influencing factors in vitro. Dent Mater 2008; 24(4):433-49.

19. Ritzberger C, Apel E, Höland W, Peschke A, Rheinberger VM. Properties and clinical application of three types of dental glass-ceramics and ceramics for CAD-CAM technologies. Dent Mater 2010; 3:3700-13.

20. Denry I, Kelly JR. State of the art of zirconia for dental applications. Dent Mater 2008; 24:299-307.

21. Taskonak B, Mecholsky Jr JJ, Anusavice KJ. Residual stresses in bilayer dental ceramics. Biomaterials 2005; 26:3235-41.

22. Encke BS, Heydecke G, Wolkewitz M, Strub JR. Results of a prospective randomized controlled trial of posterior $\mathrm{ZrSiO}$ (4)-ceramic crowns. J Oral Rehabil 2009; 36(3):226-35.

23. Nicolaisen MH, Bahrami G, Schropp L, Isidor F. Functional and Esthetic Comparison of Metal-Ceramic and All-Ceramic Posterior Three-Unit Fixed Dental Prostheses. Int J Prosthodont 2016; 29(5):473-81.

24. Makarouna M, Ullmann K, Lazarek K, Boening KW. Six-year clinical performance of lithium disilicate fixed partial dentures. Int J Prosthodont 2011; 24(3):204-6.

25. Goodacre CJ, Bernal G, Rungcharassaeng K, Kan JY. Clinical complications in fixed prosthodontics. J Prosthet Dent 2003; 90(1):31-41.

26. Walton TR. An up to 15 -year longitudinal study of 515 metal-ceramic FPDs: Part 2. Modes of failure and influence of various clinical characteristics. Int J Prosthodont 2003; 16(2):177-82

27. Esquivel-Upshaw JF, Anusavice KJ, Young H, Jones J, Gibbs C. Clinical performance of a lithia disilicate-based core ceramic for three-unit posterior FPDs. Int J Prosthodont 2004; 17(4):469-75

28. Fradeani M, D’Amelio M, Redemagni M, Corrado M. Fiveyear follow-up with Procera all-ceramic crowns. Quintessence Int 2005; 36(2):105-13.

29. Frankenberger R, Petschelt A, Kramer N. Leucite-reinforced glass ceramic inlays and onlays after six years: clinical behavior. Oper Dent 2000; 25(6):459-65.

30. Olsson KG, Furst B, Andersson B, Carlsson GE. A long-term retrospective and clinical follow-up study of In-Ceram Alumina FPDs. Int J Prosthodont 2003; 16(2):150-6.

31. Pallesen U, van Dijken JW. An 8-year evaluation of sintered ceramic and glass ceramic inlays processed by the Cerec CAD/CAM system. Eur J Oral Sci 2000; 108(3):239-46.

32. Bindl A, Mormann WH. An up to 5-year clinical evaluation of posterior in-ceram CAD/CAM core crowns. Int J Prosthodont 2002; 15(5):451-6.

33. McLaren EA, White SN. Survival of In-Ceram crowns in a private practice: a prospective clinical trial. J Prosthet Dent 2000; 83(2):216-22.

34. Suarez MJ, Lozano JF, Paz Salido M, Martinez F. Three-year clinical evaluation of In-Ceram Zirconia posterior FPDs. Int J Prosthodont 2004; 17(1):35-8.

35. Vult von Steyern P, Jonsson O, Nilner K. Five-year evaluation of posterior all-ceramic three-unit (In-Ceram) FPDs. Int J Prosthodont 2001; 14(4):379-84

36. Fradeani M, Redemagni M. An 11-year clinical evaluation of leucite-reinforced glass-ceramic crowns: a retrospective study. Quintessence Int 2002; 33(7):503-10. 
37. Naenni N, Bindl A, Sax C, Hämmerle C, Sailer I. A randomized controlled clinical trial of 3-unit posterior zirconia-ceramic fixed dental prostheses (FDP) with layered or pressed veneering ceramics: 3-year results. J Dent 2015; 43(11):136570 .

38. Anusavice KJ, Shen C, Rawls HR. Philips - Materiais dentários. Elsevier: Rio de Janeiro; 2013.

39. Conceição EN. Restaurações Estéticas: Compósitos, Cerâmicas e Implantes. Artes Médicas: São Paulo; 2005.

40. Stawarczyk B, Frevert K, Ender A, Roos M, Sener B, Wimmer T. Comparison of four monolithic zirconia materials with conventional ones: contrast ratio, grain size, four-point flexural strength and two-body wear. J Mech Behav Biomed Mater 2016; 59:128-38.

41. Johansson C, Kmet G, Rivera J, Larsson C, Vult Von Steyern P. Fracture strength of monolithic all-ceramic crowns made of high translucent yttrium oxide-stabilized zirconium dioxide compared to porcelain-veneered crowns and lithium disilicate crowns. Acta Odontol Scand 2014; 72(2):145-53.

42. Sulaiman TA, Delgado AJ, Donovan TE. Survival rate of lithium disilicate restorations at 4 years: a retro-spective study. J Prosthet Dent 2015; 114(3):364-6.

43. Wendler M, Belli R, Petschelt A, Mevec D, Harrer W, Lube $\mathrm{T}$, et al. Chairside CAD/CAM materials. Part 2: flexural strength testing. Dent Mater 2017; 33:99-109.

44. Kang SH, Chang J, Son HH. Flexural strength and microstructure of two lithium disilicate glass ceramics for CAD/ CAM restoration in the dental clinic. Restor Dent Endod 2013; 38:134-140.

45. Guess PC, Zavanelli RA, Silva NR, Bonfante EA, Coelho PG, Thompson VP. Monolithic CAD/CAM lithium disilicate versus veneered Y-TZP crowns: comparison of failure modes and reliability after fatigue. Int J Prosthodont 2010; 23(5):43442.

46. Heffernan MJ, Aquilino SA, Diaz-Arnold AM, Haselton DR, Stanford CM, Vargas MA. Relative translucency of six all-ceramic systems. Part I: core materials. J Prosthet Dent 2002; 88(1):4-9.

47. Kok P, Kleverlaan CJ, Jager N, Kuijs R, Feilzer AJ. Mechanical performance of implant-supported posterior crowns. J Prosthet Dent 2015; 114(1):59-66.

48. Baladhandayutham B, Lawson NC, Burgess JO. Fracture load of ceramic restorations after fatigue load-ing. J Prosthet Dent $2015 ; 114(2): 266-71$

49. Nordahl N, Vult von Steyern P, Larsson C. Fracture strength of ceramic monolithic crown systems of dif-ferent thickness. J Oral Sci 2015; 57(3):255-61.

50. Moscovitch M. Consecutive case series of monolithic and minimally veneered zirconia restorations on teeth and implants: up to 68 months. Int J Periodontics Restorative Dent $2015 ; 35(3): 315-23$

51. Amer R, Kurklu D, Johnston W. Effect of simulated mastication on the surface roughness of three ceramic systems. J Prosthet Dent 2015; 114(2):260-5.

52. Kim MJ, Oh SH, Kim JH, Ju SW, Seo DG, Jun SH, et al. Wear evaluation of the human enamel opposing different Y-TZP dental ceramics and other porcelains. J Dent. 2012; 40(11):979-88.

53. Rosentritt M, Preis V, Behr M, Hahnel S, Handel G, Kolbeck C. Two-body wear of dental porcelain and substructure oxide ceramics. Clin Oral Investig 2012; 16(3):935-43.

54. Janyavula S, Lawson N, Cakir D, Beck P, Ramp LC, Burgess JO. The wear of polished and glazed zirconia against enamel. J Prosthet Dent 2013; 109(1):22-9.
55. Syklawer SB, Janyavula S, Beck P, Cakir D. Wear of ceramics and enamel in artificial chewing simulator. J Dent Res 2013; 92:1902.

56. Nishioka RS. Prótese adesiva sem metal com sistema IPS Empress 2. Rev Assoc Paul Cir Dent 2002; 56(4):277-9.

57. Liu PR. A panorama of dental CAD/CAM restorative systems. Compend Contin Educ Dent 2005; 26(7):507-10.

58. Miyazaki T, Hotta Y, Kunii J, Kuriyama S, Tamaki Y. A review of dental CAD/CAM: current status and future perspectives from 20 years of experience. Dent Mater J 2009; 28(1):44-56.

59. Preis V, Weiser F, Handel G, Rosentritt M. Wear performance of monolithic dental ceramics with different surface treatments. Quintessence Int 2013; 44(5):393-405.

60. Quinn JB, Sundar V, Parry EE, Quinn GD. Comparison of edge chipping resistance of PFM and veneered zirconia specimens. Dent Mater 2010; 26(1):13-20.

61. Zhang Y, Chai H, Lee JJ, Lawn BR. Chipping resistance of graded zirconia ceramics for dental crowns. J Dent Res 2012; 91(3):311-5.

\section{Endereço para correspondência:}

Wilson da Silva Junior

Rua Pernambuco, 1464, Centro

85810-021, Cascavel, Paraná, Brasil

Telefone: (+55) (45) 9 9910-1067

E-mail: silva.wilson@outlook.com.br

Recebido: 09/08/18. Aceito: 18/09/18. 